\title{
THE KRUSKAL-KATONA THEOREM AND A CHARACTERIZATION OF SYSTEM SIGNATURES
}

\author{
ALESSANDRO D'ANDREA* AND \\ LUCA DE SANCTIS, ${ }^{* * *}$ Università degli Studi di Roma 'La Sapienza'
}

\begin{abstract}
We show how to determine if a given vector can be the signature of a system on a finite number of components and, if so, exhibit such a system in terms of its structure function. The method employs combinatorial results from the theory of (finite) simplicial complexes, and provides a full characterization of signature vectors using a theorem of Kruskal (1963) and Katona (1968). We also show how the same approach can provide new combinatorial proofs of further results, e.g. that the signature vector of a system cannot have isolated zeroes. Finally, we prove that a signature with all nonzero entries must be a uniform distribution.
\end{abstract}

Keywords: Signature; reliability; simplicial complexes

2010 Mathematics Subject Classification: Primary 60C05

Secondary $93 \mathrm{E} 03$

\section{Introduction}

The concept of a signature of a system is useful in providing knowledge of the lifetime distribution of the system in terms of its structure function and single components' lifetimes only; see [2] and [7] for a thorough introduction to the subject.

In this paper we provide a combinatorial characterization of signature vectors, which seems to be an open issue in the theory of system reliability (in [5] a complete study of systems with up to five components is provided). Our approach provides a criterion to check whether a probability vector can be a signature. The method consists of simple tests involving entries of the candidate signature, and, if the tests are positive, it constructs explicitly the structure function of a system with the required signature. If the candidate signature vector does not fulfill a certain technical requirement, the procedure yields a family of sets of components which does not have the necessary algebraic properties so that no system can have that vector as its signature.

The idea is to translate the problem in combinatorial terms, and then use a result of Kruskal [4] and Katona [3], that offers a necessary and sufficient condition for a family of sets with a certain algebraic property to exist. We show that the family of cut sets of any system enjoys such a property so that the characterization problem is eventually reduced to counting the number of cut sets of each possible cardinality.

The paper is organized as follows. In Section 2 we recall the main definitions and notions in the theory of system reliability in terms of signatures, and, in particular, the relation between the

Received 22 February 2013; revision received 28 April 2014.

* Postal address: Dipartimento di Matematica, Università degli Studi di Roma 'La Sapienza', Piazzale Aldo Moro 5, Rome, 00185, Italy.

** Email address: luca.desanctis@gmail.com 
signature and the number of cut sets of each cardinality. Section 3 is devoted to the definition of simplicial complexes and their relation with cut sets and, therefore, with the signature as well. In Section 4 we recall the statement of the Kruskal-Katona theorem for simplicial complexes. Section 5 contains the main result as a summary of the observations of the previous sections: a criterion and a procedure testing whether a vector can be the signature of some system.

We then apply techniques from the theory of simplicial complexes to our problem, which allows us to obtain more interesting results. We prove two more properties of system signatures in Section 6, namely that the signature cannot have isolated zeroes, and that the signature is uniform as soon as all of its entries are nonzero. The former results follows from [6, Theorem 2] as a consequence of the so-called increasing failure rate average property, yet here we provide a new proof of combinatorial nature. We conclude with some comments in Section 7.

\section{Review of system signatures}

In this section we recall some concepts and definitions in the theory of system reliability (see [7] for more details).

Let $\tau=\left\{\tau_{1}, \ldots, \tau_{n}\right\}$ be a set of $n \in \mathbb{N}$ binary stochastic processes, interpreted as the state, as time evolves, of the components $\left\{X_{1}, \ldots, X_{n}\right\}=X$ of a system. Each component $X_{l}$ can be either down (or broken/off) or up (or working/on), e.g. $\tau_{l}=0,1$, respectively, $l=1, \ldots, n$. We assume that all components are initially up, and when a component fails, it stays down forever, so each component $X_{l}$ has a random lifetime $T_{l}$, whose distribution is assumed to be continuous in order to avoid ties in failures. Lifetimes of components can be assumed to have the same distribution and to be independent, although exchangeability is enough. A system deploys its components according to some design architecture, and is characterized by a structure function $\phi$ that indicates whether the whole system is up or down, for any given description of the states of individual components. In other words, the system may work even if some components are broken, and, given a subset $G \subseteq X$, interpreted as the set of working components, the function

$$
\phi: 2^{X} \rightarrow\{0,1\}
$$

tells us if the system is up $(\phi(G)=1)$ or down $(\phi(G)=0)$. Common sense requires $\phi$ to be nondecreasing, which means $A \subseteq B$ implies that $\phi(A) \leq \phi(B)$, and to satisfy $\phi(\varnothing)=0$, $\phi(X)=1$.

Initially all components (hence the whole system) work, and then one at a time they fail (and stay broken) so that at some point the system stops working, say this occurs as the $l$ th failure of a components takes place. The order in which components fail is a permutation $\sigma: \mathbb{N} \rightarrow \mathbb{N}$ of the set $\{1, \ldots, n\}$, encoding that $\phi\left(\left\{X_{\sigma(l)}, \ldots, X_{\sigma(n)}\right\}\right)=1$, but $\phi\left(\left\{X_{\sigma(l+1)}, \ldots, X_{\sigma(n)}\right\}\right)=0$. We may rephrase this by saying that (for a given system $\phi$ ) one and only one breakdown index $l \in\{1, \ldots, n\}$ is associated with any given ordering of the failures (permutation) $\sigma$. Let $N_{l}(\phi)$ be the number of permutations with breakdown index $l$, i.e. such that $\phi\left(\left\{X_{\sigma(l)}, \ldots, X_{\sigma(n)}\right\}\right)=1$, but $\phi\left(\left\{X_{\sigma(l+1)}, \ldots, X_{\sigma(n)}\right\}\right)=0$. Define $N(\phi)=\left(N_{1}(\phi), \ldots, N_{n}(\phi)\right) \in \mathbb{N}^{n}$.

Definition 2.1. The system signature is the probability vector $s(\phi)=N(\phi) / n$ !, whose $l$ th entry $s_{l}(\phi)$ is the probability that the system stops working exactly as the $l$ th failure of a component takes place.

An important question arises: given a vector, how can we determine whether it is the signature of some system? If so, what is a procedure to yield an explicit system inducing that signature? 
Further questions on the distributions of zero and nonzero entries have been raised by the observations of actual systems [5]. We intend to address such questions in this paper.

In the rest of this section we will recall the standard notations for the families of sets that determine the state of the system, revealing the combinatorial nature of the signature, which allows one to study the system reliability [7] combinatorially rather than as a stochastic process.

Definition 2.2. A subset $B \subseteq X$ is called a cut set if the system cannot work when all its components are broken. A subset $G \subseteq X$ is called a path set if the system works whenever all its components work. A set of either type is said to be minimal if none of its proper subsets enjoys the same property.

As the system evolves in time, choose from the set of all total orderings of $\{1, \ldots, n\}$ the element $\sigma$ that indicates the order in which the components failed. Say $\sigma$ has breakdown index $l$, meaning that the system goes down as soon as the component $X_{\sigma(l)}$ breaks. Then (see [1]),

$$
S_{l}(\phi)=s_{1}(\phi)+\cdots+s_{l}(\phi)=\frac{1}{\left(\begin{array}{l}
n \\
l
\end{array}\right)} \sum_{B \subseteq X}^{|B|=l}(1-\phi(B)),
$$

since $\sum_{B \subseteq X}^{|B|=l}(1-\phi(B))$ counts the number of subsets $B$ of $X$ with cardinality $l$ on which the structure function takes value 0 , i.e. the cut sets of cardinality $l$. Elements of each cut set $B$ and of those of its complement $X \backslash B$ can be (separately) freely permuted, so this term is multiplied by $l !(n-l)$ !.

Definition 2.3. We will call the complement of a cut set a co-cut set. By co-path, we will indicate the complement of a path set.

Remark 2.1. From (2.1) we see that the signature depends only on the number of co-cut sets of each cardinality. Equivalently, the signature depends only on the number of cut sets, or of path sets, or of co-path sets.

Consider an ordering $\sigma$ of component failures with breakdown index $l$. This means that the set of the $l-1$ components that fail first does not contain a cut set, and that the remaining $n-l+1$ components include a path set (the system is still working at the time of the $(l-1)$ th failure) and, therefore, a minimal path set as well. We also know that the set of the $l$ components that fail first does contain a cut set (and, therefore, also a minimal cut set), and the remaining $n-l$ components do not include any path sets. The component $X_{\sigma(l)}$ giving place to the $l$ th failure belongs then to both a minimal cut set and a minimal path set. Since $\sigma$ indicates the order in which the components fail, the component $X_{\sigma(l)}$ is the common element to the minimal cut and path sets that appear in the first $l$ and last $n-l+1$ positions of the vector $\left(X_{\sigma(1)}, \ldots, X_{\sigma(n)}\right)$, respectively. This is a general fact.

Remark 2.2. Each minimal cut set intersects all minimal path sets, and the intersection consists of exactly one element. Conversely, each minimal path set intersects all minimal cut sets, and the intersection has cardinality 1 .

It is not difficult to see that the structure function is fully determined by the family of the minimal cut sets or, equivalently, by the family of the minimal path set. The system is thus completely defined by its structure function or by its family of minimal cut or path sets. This one-to-one correspondence that associates nondecreasing functions $2^{X} \rightarrow\{0,1\}$ with subsets of $2^{X}$ admitting no proper inclusions will be denoted by $\Omega$, and justifies the following notation. 
Definition 2.4. Given a structure function $\phi$, the corresponding family of minimal cut sets is $\Omega(\phi)$ and given a family $\bar{\Omega}$ of subsets of $X$ without proper inclusions, the corresponding unique structure function will be denoted by $\phi_{\bar{\Omega}}=\Omega^{-1}(\bar{\Omega})$.

\section{Simplicial complexes and cut sets}

In this section we recall some notions in the theory of simplicial complexes. A simplicial complex $K$ is a set of simplices such that any face of a simplex from $K$ is also in $K$ and so the intersection of any two simplices $\Sigma_{1}, \Sigma_{2} \in K$ is a face of both $\Sigma_{1}$ and $\Sigma_{2}$. A simplicial $d$-complex is a simplicial complex where the largest dimension of any of its simplices is $d$. The $f$-vector of a simplicial $d$-complex is the vector $\left(f_{0}, f_{1}, \ldots, f_{d}\right)$ whose $l$ th component is the number of $(l-1)$-dimensional faces in the simplicial complex, and, by convention, $f_{0}=1$ unless the complex is empty.

It is important to note that not all integral vectors can be $f$-vectors of a simplicial complex. In fact, there are constraints on the number of lower-dimensional simplices one must obtain when removing vertices from a given simplex in the complex. The Kruskal-Katona theorem provides a full characterization of $f$-vectors of simplicial complexes.

Now we want to relate the family of cut sets to the concept of simplicial complex. Let $\tilde{\Omega}$ be the family of cut sets of a system with structure function $\phi$, and consider $\mathcal{C} \equiv 2^{X} \backslash \tilde{\Omega}$, i.e. the family of co-cut sets. Then $\mathcal{C}=\phi^{-1}(0)$ is a simplicial complex. In fact, since adding an element to a cut set yields a cut set of increased cardinality, removing an element from a co-cut set yields a co-cut set of decreased cardinality: this is just the very essence of simplicial complexes. Similarly, using path sets instead of cut sets, the family of co-path sets $\mathcal{P} \equiv 2^{X} \backslash \phi^{-1}(1)=\phi^{*-1}(0)$ is also a simplicial complex (see Appendix A for the notation regarding $\left.\phi^{*}\right)$.

We will focus on cut sets only, but our considerations stay unchanged if we consider path sets instead. In simpler terms, a superset of a cut or path set is still a cut or path set, respectively. Denote by $\mathcal{C}_{l}$ and $\tilde{\Omega}_{l}$ the set of elements of $\mathcal{C}$ and $\tilde{\Omega}$, respectively, of cardinality $l$ so that $\mathcal{C}=\cup_{l} \mathcal{C}_{l}$ and $\tilde{\Omega}=\cup_{l} \tilde{\Omega}_{l}$. Clearly, $\mathcal{C}_{l} \cup \tilde{\Omega}_{l}=\left(\begin{array}{c}X \\ l\end{array}\right)$ and $\left|\mathcal{C}_{l}\right|+\left|\tilde{\Omega}_{l}\right|=\left(\begin{array}{c}n \\ l\end{array}\right)$. If $A \in \mathcal{C}_{l}$ and $x \in A$, then $A \backslash x \in \mathcal{C}_{l-1}$. Therefore, the vector $\left(\left|\mathcal{C}_{1}\right|, \ldots,\left|\mathcal{C}_{n}\right|\right)$ is the $f$-vector of the simplicial complex $\mathcal{C}$.

Knowledge of this vector is equivalent to knowledge of the family of cut sets, since $\tilde{\Omega}_{l}=$ $\left(\begin{array}{c}X \\ l\end{array}\right) \backslash \mathcal{C}_{l}$. However, as noted in Remark 2.1, the vector $\left(\left|\tilde{\Omega}_{1}\right|, \ldots,\left|\tilde{\Omega}_{n}\right|\right)$ is the nonnormalized cumulative signature whose $l$ th component coincides with $\left(\begin{array}{c}n \\ l\end{array}\right) S_{l}(\phi)=\left(\begin{array}{c}n \\ l\end{array}\right)\left(s_{1}(\phi)+\cdots+s_{l}(\phi)\right)$. Therefore, the $f$-vector of $\mathcal{C}$, with components $f_{l}=\left(\begin{array}{l}n \\ l\end{array}\right)\left(1-S_{l}\right), l=1, \ldots, n$, is trivially related to the signature. Clearly, $1-S_{l}$ equals $s_{l+1}+\cdots+s_{n}$ by definition.

In the next section we will introduce the theorem of Kruskal-Katona, which provides a characterization of $f$-vectors and, hence, of signatures.

\section{Kruskal-Katona theorem}

We recall here the Kruskal-Katona theorem, which provides a characterization of $f$-vectors of simplicial complexes, that we will later apply towards understanding system signatures. Given two integers $k \geq 0$ and $l>0$, it is known that there is a unique way to expand $k$ as a sum of binomial coefficients as

$$
k=\left(\begin{array}{c}
n_{l} \\
l
\end{array}\right)+\left(\begin{array}{c}
n_{l-1} \\
l-1
\end{array}\right)+\cdots+\left(\begin{array}{c}
n_{j} \\
j
\end{array}\right)
$$

with $n_{l}>n_{l-1}>\cdots>n_{j} \geq j \geq 1$. 
As an example, consider $n=25, l=3$. The largest integer of the form $\left(\begin{array}{c}n_{3} \\ 3\end{array}\right)$ smaller than or equal to 25 is $20=\left(\begin{array}{l}6 \\ 3\end{array}\right)$. The largest integer of the form $\left(\begin{array}{c}n_{2} \\ 2\end{array}\right)$ smaller than or equal to $5=25-20$ is $3=\left(\begin{array}{l}3 \\ 2\end{array}\right)$. The largest integer of the form $\left(\begin{array}{c}n_{1} \\ 1\end{array}\right)$ smaller than or equal to $2=5-3$ is $2=\left(\begin{array}{l}2 \\ 1\end{array}\right)$. This completes the example, since

$$
\left(\begin{array}{l}
6 \\
3
\end{array}\right)+\left(\begin{array}{l}
3 \\
2
\end{array}\right)+\left(\begin{array}{l}
2 \\
1
\end{array}\right)=20+3+2=25
$$

Note that $n_{i} \geq i$ for every $i$ so all binomial summands are necessarily positive; as a consequence, when $k=0$, the unique admissible expansion is the empty one.

Now, for the given $k$ and $l$, define

$$
k^{+}(l)=\left(\begin{array}{c}
n_{l} \\
l+1
\end{array}\right)+\left(\begin{array}{c}
n_{l-1} \\
l
\end{array}\right)+\cdots+\left(\begin{array}{c}
n_{j} \\
j+1
\end{array}\right)
$$

and

$$
k^{-}(l)=\left(\begin{array}{c}
n_{l} \\
l-1
\end{array}\right)+\left(\begin{array}{c}
n_{l-1} \\
l-2
\end{array}\right)+\cdots+\left(\begin{array}{c}
n_{j} \\
j-1
\end{array}\right)
$$

from the previous expansion. When $k=0$, this forces $k^{+}(l)=k^{-}(l)=0$.

The next proposition is a version of the Kruskal-Katona theorem and offers a minimality constraint for simplicial complexes with emphasis on the combinatorial aspects of the sets composing the complex. In fact, the term 'complex' is not even used in the terminology.

Proposition 4.1. (See [4] and [3].) Let $\underline{X}$ be a set of $n$ elements $k$ and $l$ be given integers such that

$$
1 \leq l \leq n, \quad 0 \leq k \leq\left(\begin{array}{l}
n \\
l
\end{array}\right),
$$

and let

$$
\mathcal{A}=\left\{A_{1}, \ldots, A_{k}\right\}, \quad A_{i} \subseteq \underline{X}, \quad\left|A_{i}\right|=l \quad \text { for } i=1, \ldots, k .
$$

If

$$
\mathcal{A}^{-}=\left\{B:|B|=l-1 \text {, there exists } j: B \subset A_{j}\right\}
$$

then

$$
\min _{\mathscr{A}}\left|\mathcal{A}^{-}\right|=k^{-}(l),
$$

where the minimum runs over all collections $\mathcal{A}$ of $k$ subsets of $\underline{X}$ of cardinality $l$, and $k^{-}(l)$ is defined as in (4.2).

Here and in what follows, for the original proof and a more general analysis, see [4] and [3]. From (4.3) it follows that families $\mathcal{A}$ of increasing or decreasing cardinality form a simplicial complex. The next proposition is probably the most common version of the Kruskal-Katona theorem, equivalent to Proposition 4.1, and provides a necessary and sufficient condition on the number of $l$-simplices in order for them to be induced from a complex. These numbers are the entries of the so-called $f$-vector of the complex, whose definition has been was stated in Section 3.

Proposition 4.2. (See [4] and [3].) A vector $\left(f_{0}, f_{1}, \ldots, f_{d}\right)$ is the f-vector of a simplicial $d$-complex if and only if

$$
0 \leq f_{l}^{-}(l) \leq f_{l-1}, \quad 1 \leq l \leq d .
$$


In the case of the application to a system signature with $n$ components, we will consider $d=n-1$, and any total ordering can be chosen for the system components. Choosing, at level $l$, initial segments (according to the reverse lexicographic order) of size $f_{l}$ makes the number of implied elements at level $l-1$ minimal. There is a dual maximality condition which is equivalent to (4.4),

$$
0 \leq f_{l+1} \leq f_{l}^{+}(l), \quad 0 \leq l \leq d-1 .
$$

The reverse lexicographic order simply reads the strings in reverse order, then sorts lexicographically. The advantage of considering the reverse lexicographic order is that the list of the first (according to this order) $r \in \mathbb{N}$ elements does not depend on the size of the alphabet (the size $n$ of the system, in our case).

\section{Characterization of system signatures}

In this section we sum up all observations made so far into the main result of this work. Let us start with a preliminary well-known observation, basically equivalent to what we presented in Section 3. Consider the signature vector $s$ of some system with $n$ components $\left\{X_{1}, \ldots, X_{n}\right\}$. The entry $s_{l}$ is the probability that the system fails at the $l$ th failure of a component. In other words, the $l$ th entry of the cumulative signature $S_{l}=s_{1}+\cdots+s_{l}$ is the probability that the first $l$ components that broke form a cut set. This probability is in turn nothing but the fraction of cut sets of cardinality $l$ among all subsets of $\left\{X_{1}, \ldots, X_{n}\right\}$ with cardinality $l$.

Let us see how this applies in the context of system signatures when translated in terms of simplicial sets. Recall that if we add a component to a cut set, we again obtain a cut set, i.e. all supersets of a cut set are cut sets. The algorithm that we are about to present is, in fact, the translation of the proof of the Kruskal-Katona theorem, where the role of $f$-vectors is played by the 'complement' of the cumulative signature multiplied by the number of permutations of components, roughly speaking.

Theorem 5.1. Let the probability vector $\bar{s} \in \mathbb{R}^{n}$ be the candidate signature. When $l=$ $1, \ldots, n-1$, define $f_{l}=\left(\begin{array}{l}n \\ l\end{array}\right)\left(\bar{s}_{l+1}+\cdots+\bar{s}_{n}\right)$. Then $\overline{\boldsymbol{s}}$ is the signature of a system if and only if all $f_{l}$ are nonnegative integers and they satisfy

$$
0 \leq f_{l}^{-}(l) \leq f_{l-1}, \quad 1 \leq l \leq n-1 .
$$

Proof. From the candidate signature $\overline{\boldsymbol{s}}$ we also know the nonnormalized candidate cumulative signature $\bar{S}$. Clearly, $1-\bar{S}_{l}=\bar{s}_{l+1}+\cdots+\bar{s}_{n}$ by definition. Then the number $f_{l}=\left(\begin{array}{l}n \\ l\end{array}\right)\left(1-\bar{S}_{l}\right)$ must be the number of co-cut sets of cardinality $l$, as we discussed in Section 3 , if there is some system with signature $\overline{\boldsymbol{s}}$, and is thus a nonnegative integer; note that, by definition, if $f_{l}=0$ then $f_{k}=0$ for all $k \geq l$, hence (5.1) is satisfied for all such indices.

Moreover, the family of co-cut sets of our (hypothetical) system inducing $\overline{\boldsymbol{s}}$, forms a simplicial complex whose $f$-vector equals $\left(1, f_{1}, \ldots, f_{n-1}\right)$. The Kruskal-Katona theorem provides a test to check whether this vector can actually be the $f$-vector of a simplicial complex. The test consists of (5.1), as explained in Proposition 4.2.

Example 5.1. Consider the probability vector

$$
\bar{s}=\left(s_{1}, s_{2}, s_{3}, s_{4}, s_{5}\right)=\left(0, \frac{3}{10}, \frac{2}{5}, \frac{3}{10}, 0\right) .
$$

The corresponding vector is $\boldsymbol{f}=\left(f_{0}, f_{1}, f_{2}, f_{3}, f_{4}\right)=(1,5,7,3,0)$. We have

$$
f_{1}^{-}(1)=1, \quad f_{2}^{-}(2)=5, \quad f_{3}^{-}(3)=6, \quad f_{4}^{-}(4)=0 .
$$


For instance, $f_{2}=7$ can be written as $\left(\begin{array}{l}4 \\ 2\end{array}\right)+\left(\begin{array}{l}1 \\ 1\end{array}\right)$, hence $f_{2}^{-}(2)$ equals $\left(\begin{array}{l}4 \\ 1\end{array}\right)+\left(\begin{array}{l}1 \\ 0\end{array}\right)=5$. We see that (5.1) is satisfied, as 1, 5, 6, 0 are not greater than, respectively, 1, 5, 7, 3, and we conclude that $\bar{s}$ is a signature vector.

Sometimes an equivalent procedure might be easier to perform, especially for small systems. The following is such a procedure. Let $\bar{N} \equiv n ! \bar{s} \in \mathbb{N}^{n}$.

(i) For each $l=1, \ldots, n$, sort in lexicographic order the subsets of $\{1, \ldots, n\}$ of cardinality $l$.

(ii) Take the family $\Omega^{l}$ of the first $\left(\bar{N}_{1}+\cdots+\bar{N}_{l}\right) /(n-l) ! l !=\left(\bar{s}_{1}+\cdots+\bar{s}_{l}\right)\left(\begin{array}{l}n \\ l\end{array}\right)$ subsets, with respect to the lexicographic order.

(iii) Take the union $\cup_{l=1}^{n} \Omega^{l}$ of all the $\Omega^{l}, l=1, \ldots, n$, and extract the minimal family $\bar{\Omega}$.

(iv) The function $\phi_{\bar{\Omega}}$ is the structure function of a system $X$ with $n$ components and signature $\bar{s}$.

Now considering Proposition 4.1, the arguments of Theorem 5.1 also prove the following test.

Criterion 5.1. The family $\Omega^{l+1}$ should contain all the supersets (of cardinality $l+1$ ) of at least one element from $\Omega^{l}$. If this is not the case then the vector $\bar{s}$ cannot be the signature of a system, since $\cup_{h} \Omega^{l}$ is not a simplicial complex.

This criterion is equivalent to Theorem 5.1, and the algorithm we presented is simply the Kruskal-Katona algorithm adjusted to work directly with the candidate nonnormalized cumulative signature as opposed to its 'complementary' vector with components $\left(\begin{array}{l}n \\ l\end{array}\right)\left(1-\bar{S}_{l}(\phi)\right)$, $l=1, \ldots, n$. This is the reason why we sort strings lexicographically, because the collection $\mathcal{C}$ is a simplicial complex, as opposed to $\tilde{\Omega}$. So, instead of taking, as in the original KruskalKatona algorithm, initial segments in each $\mathcal{C}_{l}$ according to reverse lexicographic order, we take final segments, i.e. initial segments according to the reverse ordering, which is the lexicographic order, in each $\tilde{\Omega}_{l}$.

We want to show that this second algorithm can be fairly fast in an explicit detailed example. The reader may want to compare this example with the systematic study of small systems in [5].

Example 5.2. Consider the vector $\left(0, \frac{3}{10}, \frac{2}{5}, \frac{3}{10}, 0\right)$. We pass easily to the nonnormalized vector $(0,36,48,36,0)$ by multiplying by 5 .

Start with $l=1$. We must take the first $\frac{0}{1 ! 4 !}=0$ singletons.

Take $l=2$. We must take the (lexicographically) first $\frac{36}{2 ! 3 !}=3$ subsets with two elements. They are $\{1,2\},\{1,3\}$, and $\{1,4\}$.

Take $l=3$. We must take the first $\frac{36+48}{3 ! 2 !}=\frac{84}{12}=7$ subsets with three elements. These are $\{1,2,3\},\{1,2,4\},\{1,2,5\},\{1,3,4\},\{1,3,5\},\{1,4,5\}$, and $\{2,3,4\}$.

Take $l=4$. We must take the first $\frac{36+48+36}{4 ! 1 !}=\frac{120}{24}=5$ subsets with four elements. These are $\{1,2,3,4\},\{1,2,3,5\},\{1,2,4,5\},\{1,3,4,5\}$, and $\{2,3,4,5\}$.

Take $l=5$. We must take the first $\frac{120}{120}=1$ subsets with five elements. This is $\{1,2,3,4,5\}$.

From these subsets we must extract a minimal family. It is not difficult to obtain $\bar{\Omega}=$ $\{\{1,2\},\{1,3\},\{1,4\},\{2,3,4\}\}$. In fact, all other listed sets are supersets of these four selected sets.

Even calculating $\bar{\Omega}$ can be automatized: in general, if $f_{l}$ subsets are missing at level $l$, one should take, at level $l+1$, all subsets that come after the first $\left(\begin{array}{c}n \\ l+1\end{array}\right)-f_{l}^{+}(l)$. Let us perform this computation explicitly in the above example: since

$$
\left(f_{0}, f_{1}, f_{2}, f_{3}, f_{4}\right)=(1,5,7,3,0)
$$


we obtain

$$
f_{1}^{+}(1)=10, \quad f_{2}^{+}(2)=4, \quad f_{3}^{+}(3)=0, \quad f_{4}^{+}(4)=0 .
$$

Then we can recover $\bar{\Omega}$ by omitting the (lexicographically) first $\left(\begin{array}{c}n \\ l+1\end{array}\right)-f_{l}^{+}(l)$ subsets of cardinality $l+1$, from the above lists. This means we should omit the first $0=\left(\begin{array}{l}5 \\ 2\end{array}\right)-10$ subsets of cardinality 2 , and take the remaining three; omit the first $6=\left(\begin{array}{l}5 \\ 3\end{array}\right)-4$ subsets of cardinality 3 , and take just the last one; and then omit all subsets of higher cardinality. We obtain, as above, $\bar{\Omega}=\{\{1,2\},\{1,3\},\{1,4\},\{2,3,4\}\}$.

This fully determines the system, and we can use the definition of minimal cut sets to determine the structure function $\phi_{\bar{\Omega}}$ and verify that $N\left(\phi_{\bar{\Omega}}\right)=(0,36,48,36,0)$.

\section{Two more properties of signatures}

In this section we provide two properties of system signatures.

\subsection{Signature vectors cannot have isolated zeroes}

In the next theorem we provide a new combinatorial proof of a previously established property of signatures. Namely, system signature cannot have isolated zeroes, which follows from [6, Theorem 2].

We use the same notation as in the previous section: $X$ is the set of system components, $\tilde{\Omega}$ is the family of cut sets, and $\tilde{\Omega}_{l}$ is the family of all cut sets with cardinality $l$. Recall that $\left|\tilde{\Omega}_{l}\right|=\left(\begin{array}{l}n \\ l\end{array}\right) S_{l}(\phi)$, where $S_{l}(\phi)=s_{1}(\phi)+\cdots+s_{l}(\phi)$ is the cumulative signature, if $s(\phi)$ is the signature vector of a system with structure function $\phi$. Should $s_{l}(\phi)$ be 0 , we would have $S_{l}(\phi)=S_{l-1}(\phi)$. Therefore, the condition

$$
\frac{\left|\tilde{\Omega}_{l}\right|}{\left(\begin{array}{c}
n \\
l
\end{array}\right)}=\frac{\left|\tilde{\Omega}_{l-1}\right|}{\left(\begin{array}{c}
n \\
l-1
\end{array}\right)}
$$

is equivalent to the vanishing of the $l$ th entry in the signature. We want to show that this cannot occur unless all subsequent (or preceding, by duality) entries are all 0.

Theorem 6.1. Let $C_{l}$ be the number of cut sets of cardinality $l$. If

$$
C_{l-1} \neq 0\left(\begin{array}{c}
n \\
l-1
\end{array}\right)
$$

then

$$
\frac{C_{l-1}}{\left(\begin{array}{c}
n \\
l-1
\end{array}\right)}<\frac{C_{l}}{\left(\begin{array}{c}
n \\
l
\end{array}\right)} .
$$

Proof. We know from the previous section that $\mathcal{C}_{l}=\left(\begin{array}{c}X \\ l\end{array}\right) \backslash \tilde{\Omega}_{l}$ is a simplicial set so the Kruskal-Katona theorem applies. Therefore. we have

$$
\left|\mathcal{C}_{l}\right|=\left(\begin{array}{c}
c_{l} \\
l
\end{array}\right)+\left(\begin{array}{c}
c_{l-1} \\
l-1
\end{array}\right)+\cdots,
$$

for suitable integers $n \geq c_{l}>c_{l-1}>\cdots$

We can assume that $n>a_{l}$, since $\left|\mathcal{C}_{l}\right| \neq\left(\begin{array}{l}n \\ l\end{array}\right)$. Our aim is to show that

$$
1-\frac{\left|\mathcal{C}_{l}\right|}{\left(\begin{array}{l}
n \\
l
\end{array}\right)}>1-\frac{\left|\mathcal{C}_{l-1}\right|}{\left(\begin{array}{c}
n \\
l-1
\end{array}\right)}
$$


or, equivalently,

$$
\frac{\left|\mathcal{C}_{l}\right|}{\left(\begin{array}{c}
n \\
l
\end{array}\right)}<\frac{\left|\mathcal{C}_{l-1}\right|}{\left(\begin{array}{c}
n \\
l-1
\end{array}\right)}
$$

In order to make the notation lighter, let us put $\boldsymbol{C}_{l}=\left|\mathcal{C}_{l}\right|$ so that, according to the notation of the previous section,

$$
\boldsymbol{C}_{l}^{-}=\left(\begin{array}{c}
c_{l} \\
l-1
\end{array}\right)+\left(\begin{array}{c}
c_{l-1} \\
l-2
\end{array}\right)+\cdots
$$

and

$$
\boldsymbol{C}_{l-1} \geq \boldsymbol{C}_{l}^{-}
$$

Hence, we have only to prove that

$$
\frac{\boldsymbol{C}_{l}}{\left(\begin{array}{c}
n \\
l
\end{array}\right)}<\frac{\boldsymbol{C}_{l}^{-}}{\left(\begin{array}{c}
n \\
l-1
\end{array}\right)}
$$

which is the same as

$$
\frac{\boldsymbol{C}_{l}^{-}}{\boldsymbol{C}_{l}}>\frac{\left(\begin{array}{c}
n \\
l-1
\end{array}\right)}{\left(\begin{array}{l}
n \\
l
\end{array}\right)}
$$

In other words, we want to show that the function $\boldsymbol{C}_{l} \mapsto \boldsymbol{C}_{l}^{-} / \boldsymbol{C}_{l}, \boldsymbol{C}_{l}>0$, takes its only minimum at $\boldsymbol{C}_{l}=\left(\begin{array}{l}n \\ l\end{array}\right)$. We now use Lemma 6.1 below, the proof of which we postpone for the sake of clarity, with

$$
\begin{aligned}
& a=\left(\begin{array}{c}
c_{l} \\
l-1
\end{array}\right), \quad a^{*}=\left(\begin{array}{c}
c_{l} \\
l-2
\end{array}\right), \quad a^{\prime}=\left(\begin{array}{c}
c_{l-1} \\
l-2
\end{array}\right)+\cdots, \\
& b=\left(\begin{array}{c}
c_{l} \\
l
\end{array}\right), \quad b^{*}=\left(\begin{array}{c}
c_{l} \\
l-1
\end{array}\right), \quad b^{\prime}=\left(\begin{array}{c}
c_{l-1} \\
l-1
\end{array}\right)+\cdots \text {. }
\end{aligned}
$$

By assumption, $a^{\prime} / b^{\prime}>a^{*} / b^{*}$. Moreover, $b^{*}>b^{\prime}$ and $a^{*} \geq a^{\prime}$. Since $a / b=l /\left(c_{l}-l+1\right)$ and $a^{*} / b^{*}=(l-1) /\left(c_{l}-l+2\right)$, then $a / b>a^{*} / b^{*}$. Lemma 6.1 yields

$$
\frac{\boldsymbol{C}_{l}^{-}}{\boldsymbol{C}_{l}}=\frac{a+a^{\prime}}{b+b^{\prime}}>\frac{a+a^{*}}{b+b^{*}}=\frac{\left(\begin{array}{c}
c_{l} \\
l-1
\end{array}\right)+\left(\begin{array}{c}
c_{l} \\
l-2
\end{array}\right)}{\left(\begin{array}{c}
c_{l} \\
l
\end{array}\right)+\left(\begin{array}{c}
c_{l} \\
l-1
\end{array}\right)}=\frac{\left(\begin{array}{c}
c_{l+1} \\
l-1
\end{array}\right)}{\left(\begin{array}{c}
c_{l+1} \\
l
\end{array}\right)} \geq \frac{\left(\begin{array}{c}
n \\
l-1
\end{array}\right)}{\left(\begin{array}{c}
n \\
l
\end{array}\right)},
$$

which concludes the proof.

We are left with proving only the following easy lemma.

Lemma 6.1. Let $a, a^{\prime}, a^{*}, b, b^{\prime}$, and $b^{*}$ be positive integers such that $a^{*} \geq a^{\prime}, b^{*}>b^{\prime}$. If $a / b, a^{\prime} / b^{\prime}>a^{*} / b^{*}$, then

$$
\frac{a+a^{\prime}}{\left(b+b^{\prime}\right.}>\frac{a+a^{*}}{b+b^{*}} .
$$

Proof. It is well known that the mediant $(r+t) /(s+u)$ of any two given fractions $r / s$ and $t / u$, lies in-between, provided that $r, s, t, u>0$. Let us use this fact with $a / b$ and $\left(a^{*}-a^{\prime}\right) /\left(b^{*}-b^{\prime}\right)$ to obtain

$$
\frac{a^{*}-a^{\prime}}{b^{*}-b^{\prime}}<\frac{a^{*}}{b^{*}}<\frac{a^{\prime}}{b^{\prime}} .
$$

Therefore,

$$
\frac{a}{b}>\frac{a^{*}}{b^{*}}>\frac{a^{*}-a^{\prime}}{b^{*}-b^{\prime}}
$$


Now, both $a / b$ and $a^{\prime} / b^{\prime}$ are strictly larger than $\left(a^{*}-a^{\prime}\right) /\left(b^{*}-b^{\prime}\right)$, and, hence, so is $\left(a+a^{\prime}\right) /\left(b+b^{\prime}\right)$. Then

$$
\frac{a+a^{\prime}}{b+b^{\prime}}>\frac{a+a^{\prime}+a^{*}-a^{\prime}}{b+b^{\prime}+b^{*}-b^{\prime}}=\frac{a+a^{*}}{b+b^{*}}
$$

and the proof follows.

\subsection{Singleton cut sets correspond to uniform signatures}

Let us conclude with a final observation for which we use the notation presented in Appendix A. The signatures we have computed so far, and almost all those appearing in the literature, have initial or final entry equal to zero. An exception is the signature of a system with structure function $\phi_{1}$ associated with a minimal family of minimal cut sets consisting of the only subset $\Omega_{1}=\left\{X_{1}\right\}$. This is no coincidence. So the following result holds.

Theorem 6.2. For any system, if both the first and last entry of the signature differ from zero, then all entries coincide (and equal the inverse of the size of the system).

Proof. If both the first and last entry of the signature are different from zero, then, if $\phi$ is the structure function, both $\Omega(\phi)$ and $\Omega\left(\phi^{*}\right)$ contain a singleton, say $\left\{X_{1}\right\}$. Now all elements of $\Omega\left(\phi^{*}\right)$ must intersect all elements of $\Omega(\phi)$, and, therefore, they all contain $X_{1}$. For the same reason, all elements of $\Omega(\phi)$ contain $X_{1}$ so the only minimal cut set is $\Omega(\phi)=\left\{\left\{X_{1}\right\}\right\}$. This means that $\phi=\phi_{1}$ and we know that $s\left(\phi_{1}\right)$ is the uniform distribution (over the components of the system).

\section{Conclusions and outlook}

We have employed results obtained in the context of simplicial complexes to address questions in the theory of system reliability with a particular focus on system signatures. We have introduced a procedure, making use of the celebrated Kruskal-Katona theorem, that checks if a given probability vector can be a system signature, and, in this case, constructs a system with that signature. This completely characterizes the set of possible system signatures.

We have proved three properties of system signatures that followed observations in many numerical studies carried out in the literature (see, e.g. [5]). Namely, we have showed in a new combinatorial way that no isolated zeroes may appear in the signature vector and that the only signature with first and last component both different from zero is the uniform one. Further applications can be given, for instance about the (partial) unimodal property of the signature, and we plan on reporting on some of them soon.

\section{Appendix A. Duality of systems}

Let us recall a definition that is needed only in order to introduce a notation that is used in Section 6.2.

Definition A.1. Given a system with structure function $\phi$, the dual system has structure function $\phi^{*}$,

$$
\phi^{*}(A)=1-\phi(X \backslash A) \text { for all } A \subseteq X .
$$

Let us study some elementary families of cut sets. Given a family $\bar{\Omega}$ of subsets of $X$ without proper inclusions, and recalling Definition 2.4 , we may define its dual family by

$$
\bar{\Omega}^{*}=\Omega\left(\phi_{\bar{\Omega}}^{*}\right)
$$


independently of whether it is interpreted as a family of cut or path sets. The family of minimal cut sets of a system is also the family of the minimal path sets of the dual system (and vice versa, because duality is an involution). Duality is in essence the relation between minimal cut sets and minimal path sets, which ultimately consists of a time reversal (reading the signature in reverse order). Translated in terms of structure functions, this means that

$$
\phi_{\bar{\Omega}^{*}}=\phi_{\bar{\Omega}}^{*} .
$$

Now we can learn how to find dual families of minimal cut or path sets. Let us introduce a basic example. If, for some positive integer $h<n$, we choose $\Omega_{h}=\left\{\left\{X_{1}\right\},\left\{X_{2}\right\}, \ldots,\left\{X_{h}\right\}\right\}$ then $\Omega_{h}^{*}=\left\{\left\{X_{1}, X_{2}, \ldots, X_{h}\right\}\right\}$. This is the special case of series-parallel duality. Now let $\phi_{h}=\phi_{\Omega_{h}}$. Using for simplicity the unnormalized signature $N(\phi)=n ! s(\phi)$, it is immediate to recognize that

$$
N_{i}\left(\phi_{h}\right)=(n-h) ! h !\left(\begin{array}{l}
n-i \\
h-1
\end{array}\right), \quad i<n-h+1
$$

and

$$
N_{i}\left(\phi_{h}^{*}\right)=(n-h) ! h !\left(\begin{array}{l}
i-1 \\
h-1
\end{array}\right), \quad i>h .
$$

\section{Acknowledgements}

The authors would like to thank Mario Marietti for pointing out that a former characterization we had produced was equivalent to the one provided by Kruskal and Katona. The authors express their gratitude to Fabio Spizzichino for suggesting the problem of characterizing signatures. Alessandro D'Andrea was partially supported by 'La Sapienza' Ateneo fundings. Luca De Sanctis was partially supported by research funds of C. De Concini. The authors thank the anonymous referee for pointing out reference [6].

\section{References}

[1] Boland, P. J. (2001). Signatures of indirect majority systems. J. Appl. Prob. 38, 597-603.

[2] Gertsbakh, I. (2000). Reliability Theory. With Applications to Preventive Maintenance. Springer, Berlin.

[3] Katona, G. (1968). A theorem of finite sets. In Theory of Graphs, Academic Press, New York, pp. 187-207.

[4] Kruskal, J. B. (1963). The number of simplices in a complex, In Mathematical Optimization Techniques, University of California Press, Berkeley, pp. 251-278.

[5] Navarro, J. and Rubio, R. (2010). Computations of signatures of coherent systems with five components. Comm. Statist. Simul. Comput. 39, 68-84.

[6] Ross, S. M., Shahshahani, M. And Weiss, G. (1980). On the number of component failures in systems whose component lives are exchangeable. Math. Operat. Res. 5, 358-365.

[7] Samaniego, F. J. (2007). System Signatures and Their Applications in Engineering Reliability, (Internat. Ser. Operat. Res. Manag. Sci. 110). Springer, New York. 